\title{
Namens-, Sach- und Stellenregister
}

Bezeichnungen von Mythen (mythischen STOFFEN) sind in KAPITÄLCHEN gesetzt, während Text-Bezeichnungen kursiviert sind.

A

Abaris 484

Abbild 56

Abendrot 235

Abzu 162, 573f., 582

- qereb Apsî (das Innere des Abzu) 184

Accius

- Philoctet 419-21

Achilleus 41, 237, $247 f$.

Adonis 235

Aeneas 239, 473, 573

Aesacos 14

Äther 224, 239, 244, 252f., 257

Ätna 249

aga-Krone Siehe Numinose Machtmittel

Agamemnon 41, 237, 249

Aglauros 239

Aia-Insel (der Kirke) 252

Aias 472

Aidos 251

Aietes 237f.

Aigis $26,33,41,461,487 f$.

Aischylos (Ps.-Aischylos)

- Der gefesselte Prometheus 417-22, 424, 439

- fr. 281a Radt 251

- Prometheus-Trilogie 417

Aisopos

- Fabulae 90242

Alexander der Große 255

Alkaios

- fr. 338 Lobel/Page 245

Alkinoos 225

Alkmene 226

Allophanie 227

Alope 226

Ama-ušumgal-ana 590, Siehe auch

Theophanie

- von An im Himmel erschaffen 559
Ammianus Marcellinus

$$
\text { - 22,9,7 } 242
$$

Amun 199, 211

Amun-Re 564

An (Himmelsgott) 60-61, 77f., 563, 579

- An und das Himmelsinnere 71-73

- Tempelgründung 113-17

- Töchter von Anu 171, 180, 182

an gal karede / Innana und An

- Hochzeitsnacht von Innana und An 28788

- Innana erschafft Tag und Nacht 297

- Innanas Austrinken des Grenzflusses 293

- Transfer des E-ana in die Unterwelt 294

- Verankerung des E-ana erschafft die Erde 295, 298

- Verlauf gemäß Innanas Plan 276

- verschiedene Perspektiven der Protagonisten 285-86

- Z. 27-30 298-99

- Z. 44283

- Z. 163-165 278

Analepse 14

Anchises 235, 244

ancile (Kultschild) 241, 567, 573, 577, 584

ancilia (Kultschilde) 459, 511, 577, 584

Andromache 250

Angst 578f.

Anstand 251

Anthologia Graeca

$-5,31227$

$-5,64,1$ f 245

Antisthenes

- Odysseus 3459

Aphrodite 232ff., 243, 250

Apis-Stier 234

Apollodoros

$-1,19230 f$.

$-1,43252$

$-1,45429-30$ 
$-1,47245$

$-2,34227$

$-3,21 \mathrm{f} 478$

$-3,24 \mathrm{f} 250$

$-3,25239,250$

$-3,138470$

- 3,142-145 460

$-3,143462,487,584$

- 3,143-145 241

$-3,145469,473,487$

$-3,169240$

$-3,188$ f 232

$-5,22472$

$-6,26242$

Apollon 473, 478f.

- und Daphne $13 \mathrm{f}$.

- und die Argonauten 236, 247

- und Herakles 251

- und Orestes 242

Apollonios von Rhodos

- Argonautica

$1,547 \mathrm{f} 225$

1,916470

2,286 f 239

3,158-163 232

3,584-588 238

4,640-642 236

4,1694-1700 246

4,1701-1713 247

4,1706 f 237

Ares 48, 247, 250

Argonauten 237, $246 \mathrm{f}$.

Aristophanes

- Aves 554-559 226

Aristophon PCG fr. 11232

Aristoteles

- De caelo 221

Arktinos 43, 482

Arrianos

- Anabasis 1,4,6-8 255

Artemis 241, 468, 473

Asalluhi 580

Asios 483

Astarte 202f., 233

Astarte und das Meer

- Kol. 2, Z. x+18-Kol. 2, Z. x+19 202

Asterië 228
Asterix 256

Ate 229, 460, 462, 474f., 579

Athamas 237

Athen 242,458

Athene 241, 458, 461, 471f., 482, 485, 488, 567,583

- als Botengottheit 237, 257

- Athena Ergane 489

- Athena Polias 458, 489

- und Chryse 26, 32, 41, 43

- und die Aigis 33

- und die Großen Götter 32, 36

- und Hephaistos 231

- und Herakles 237, 251

- und Kadmos 250

- und Odysseus 237

- und Pallas 41

- und Tydeus 238

Atlas 221, 470

Atrahasīs-Epos Siehe Babylonische Sintfluterzählung

Atreus 250

Augustus 14

Aura 232

Ausgangspunkte des Himmelstransfers 54748

- Äther 547

- Himmel, allgemein 547

- Himmelsfundament 547

- Himmelsinneres 547

- höchster Teil 547

- Höhepunkt des Himmels 547

- Mitte des Himmels 547

- Vorderseite des Himmels 547

- Zikkurat des Himmels 547

\section{B}

Baal 208, 211

Babu 77, Siehe Theophanie

Babylonian Poem of the Righteous Sufferer

Siehe Ludlul bēl nēmeqi

Babylonische Sintfluterzählung 178

Baebius

- 111-130 494

Barton-Zylinder 253 
Bastarner 256

Bellerophon 236

Beredter Bauer

- P. Berlin P 3023 + P. Amherst 1, Version

B1, 142-152; P. Ramesseum A = P.

Berlin P 10499, recto, Version R, 22, 723, 2196

Beroë 239

Beschwörung bei der Geburt

- AS 16, 287 f: ii 30172

- AuOr Suppl. 23, 20: 3’ 172

- BAM 248: iii 25 // AMT 67/1: iii 19172

- Iraq 31, pl. V f: r. 59172

Beschwörung gegen den Namtar-Dämon

- CUSAS 32, 12: i 26' 165

Beschwörung gegen einen böswilligen Eid

- KAR 165: r. 5167

- Šurpu Serie

VII $1 \mathrm{f} 164$

VII $3 f 168$

Beschwörung gegen Fieber

- AuOr Suppl. 23, 14: 1168

- AuOr Suppl. 23, 14: 1-10 570

- Ugaritica 5, 17: r. 20' 168

- Ugaritica 5, 17: r 20'-27' 570

Beschwörung gegen Hexerei

- AMD 8/1, pl. 31: i 13'-16'//TCL 6, 49: 1618//KAL 2, 36: v 31'-33' 167

- Maqlû

III 31-33 173

III 34-39 171

- PBS 10/2, 18: 25'-r 24' // AMD 8/1, pl. 5458: r 1'-58' 570

- PBS 10/2, 18: 39' // AMD 8/1, pl. 54-58: 9' 174

- SpTU 4, 140: r. 15' // AMD 8/1, pl. 44 f: 9' f 172

Beschwörung gegen Hexerei, kanonisch Maqlû III/b 570

Beschwörung gegen Kollaps

- Ugaritica 5, 17: r. 12' 170

Beschwörung gegen Lamaštu

- BIN 4, 126: 10-13 174

- LKU 32: 12167

- MC 17, 443 f: 13176

Beschwörung gegen Lamaštu (?)

- AS 16, $287 \mathrm{f}$ : iv 21-23 175
Beschwörung gegen maškadu-Krankheit

- AMD 14, 191: 9165

- AS 16, 287 f: ii 1169

- AS 16, 287 f: ii 1-11 570

- BAM 182: 14' 169

- BAM 182: 14'-17' 570

- CT 23, 2-4: r 14-18 // CT 23, 5-14: iii 37-40 570

- CT 23, 2-4: r. 15 // CT 23, 5-14: iii 37170

- OECT 6, 23: 4'-12' 570

- OECT 6, 23: 5' 170

- STT 136: iv 17-20 570

- STT 136: iv 18169

Beschwörung gegen Samana

- CM 10, 76 f: 1-7 177

- STT 178: 15-18 // AMT 61, 7: r. 1'-4' 177

Beschwörung gegen Schlangen

- PBS 1/2, 131: 4-6 163

Beschwörung gegen Skorpione

- YOS 11, 37: 2 f 162

Beschwörung gegen Utukku-Dämonen

- CT 51, 142: 11 // JCS 31, 218 f: 9167

Beschwörung gegen verschiedene Krankheiten

- BAM 543: iv 26’ 169

- JCS 9, 9:10 166

- JCS 9, 10: 10 f 168

- Muššu'u Serie V/d 25168

- RA 88, 161: r. 11' 166

- Semitica 61, 13 f: 6 f 165

- STT 136: iii 34' f // JNES 49, 300: 9' f 170

- Ugaritica 5, 17: 20 f 166

- YOS 11, 8: 5169

Beschwörung gegen verschiedene Übel

- BAM 543: iv 24'-41' 570

- JCS 9, 9: 10569

- JCS 9, 10: $10 f 570$

Bestattung 603

Bewässerung 574

Bibel

- Apg 19,35 242

- Ex 8,2 213

- Ex 19,18.20 573

- Ex 34,5 573

- Jakobs Leiter 182

Blitz 205f., 213f., 244f., 256, 518-23

Blumenberg 579 
Blut 200, 213, 244, 253

Böse numinose Mächte Siehe Numinoses

Bogen 251

Boreas-Söhne 239

Borysthenes 252

Briseïs 41

Byzanz 459

C

Cassius Dio

$$
\text { - 71,8-10 } 205
$$

Catena aurea 227

Ceres Siehe Demeter

Ceyx und Alcyone 14

Chaironeia 250

Chaos 247, 255

Chryse 26, 33, 41f., 44, 481, 583, 585

Chryseïs 41

Chryses 41

Cicero 225

- De haruspicum responso 62225

- In Verrem 2,5,187 242

- Tusculanae disputationes 2,23 420-21

Claudius Claudianus

- Panegyricus de quarto consulato Honorii 444

Cornutus 436-38, 444, 447

$-18,2247$

$-19,3231$

$-20,8457$

\section{D}

Dämonen 189f., 193, 196f., 202, 215, 576, 579

Daidalos 236, 242

Danaë 226

Daphne $13 f$.

Dardanos 26, 33, 42f., 470ff., 481f., 583, 585

Dareios 245

Delos 228

Delphisches Orakel 48, 478

Demeter (Ceres) 242, 251, 260

Der Beistand der Isis, Krugtexte

- Krug B, Z. 19-21 203
Diadem 204, 252

Die Rache der Isis, P. Saqqara dem. 1, 2

- Kol. 6, Z. 18-20 200

ঠıтєเท́ৎ 467

Dike 251, 573

Diodoros

$-4,14,3251$

$-5,47471$

Diomedes 458

Dion Chrysostomos

- 11,106 249

Dionysios von Halikarnassos

- Antiquitates Romanae

1,33,1 481

$1,61,1471$

$1,67,1.3481$

$1,61,1-4481$

1,68 f 459

$1,68,1-4481$

$1,68,326,36,46,481$

$1,68,432,458$

$1,69,1-4481$

$1,69,3482,486$

$2,66,5481$

2,71 531-33

Dionysos 224, 232, 237, 239f., 242

Donner 205, 208, 211, 213f., 244f., 256

Dromos 201

Dynastische Chronik

- doppelter Transfer des Königtums vom Himmel auf die Erde 374

E

E-ana $580 \mathrm{ff}$.

Ei 233, 259

Eisen 244

Eisen (Meteoriteisen) $193 \mathrm{ff}$.

Elektra 461, 469ff., 477, 481

Endymion 235

Enki 573f., 582

Enkis Fahrt nach Nippur 574

Enkis Tempelstiftung 574

Enlil 77

Enlil und Ninlil 574

Enlil/Ellil 564 
Enmerkara 83-84, 85, 602, Siehe Lugalbanda, Siehe Theophanie

- himmlische Herkunft 559

Enmerkara und Ensuhkešana

- Inthronisation durch vier Rituale 592

- Rituale 592-94

Entrückung 473

Eos 235

- als Abendrot 236

- als Morgenröte 236

Epaphos 234

Ephesos 242

Eratosthenes

$-13232$

$-3334$

Erde $74-76$

Erechtheus 19, 25, 231

Erichthonios 231, 472

Eridu 574,582

Erinyen 234, 237, 242, 253

Eros 232, 235, 567

Erste Hethitische Heirat Ramses' II.

- Abu Simbel, Großer Tempel, Fassade, Stele Jahr 34, Z. 36-38 209

Erster Setna-Roman, P. CG Cairo 30692

- Kol. 3, Z. 12 f 201

- Kol. 3, Z. 12-15 201

- Kol. 4, Z. 7 f 196

Erzählstoff 12f., 17, 59, 76

- Aufbau 18f., 23

- mythischer Erzählstoff 59, 115-16

- Stoffwolke 38

- Unvollständigkeit 29

Etana 563, 565, 567

- ikonographische Wiedergabe des ETANASTOFFS 320

Etana-Epos Siehe Herrschaftskonzepte, Königtum

Eteokles 238

Etymologicum Magnum 231, 247, 467

Eumolpos 51

Euphrat 233, 259

Euripides

- Bacchae

1078224

- Chrysippos fr. 839,1-7 253

- Iphigenia Aulidensis
1036-1079 240

- Iphigenia Taurica

77-92 241

$977 \mathrm{f} 468$

- Medea

$954 \mathrm{f} 252$

$1321 \mathrm{f} 251$

- Phoenissae

822 f 239

Eurymedon 260

Eurynome 229

Eurystheus 229, 474

Eustathios

- ad Hom. Od. 5,294 246

Ezinam Siehe Theophanie

$\mathbf{F}$

Familiengeschichte des Petese, P. Rylands 9

- Kol. 24, Z. 1 f 199

Feuer 247, 581f., 602ff.

Firmicus Maternus

$-15,1 \mathrm{f} 484$

Flügel 570

Flügelschuhe 570

Flut, Sintflut, Überschwemmung 205, 207f., Siehe auch Mythische Stoffschemata, Sintflut-Mythos

Fruchtbarkeit 258, 577f.

Fulgentius

- Mitologiarum liber 2,6 447

\section{G}

Gaia 234, 253f., 260

Gallier 256

Gerechtigkeit 572f., 576

Geschichte mit Bes

- Kol. x+3, Z. 24 ff. 196

Getreideanbau 574

Gewichte 243

Gewitter 253, 256

Giganten 234, 240, 253, 482

Gilgameš-Epos

- XI 158183 
Götter

- als Bergbewohner 257

- Astralgottheiten 564

- aus dem Himmel 225

- bei Hochzeiten 239

- bei Kriegen 240

- Große Götter (von Samothrake) 26, 32 , 36,42

- Hochgötter 563

- kommen vom Himmel herab 553

Götterbild 576f., 583f.

- himmlische Herkunft 241-42, 554

Götterversammlung 226, 231, 238

Göttlich-naturhafte Mächte 560, Siehe Theophanie

Gottkönige, ägyptische 564

\section{H}

Hagel 205, 256

Halsband 250

Hannibal 244

Harmonia 239, 250

Harpyien 239

Hathor 189, 197f., 200

Heilige Hochzeit 577, 590

Hekate 242

Hektor 225, 248, 250

Helena 458

Helios 215f., 251, 564, 570

Helle 236

Henna 242

Hephaisteion 232

Hephaistos 222, 229, 248ff., 259ff.

- und Athene 231, 425

- und Herakles 251

- und Lemnos 498

- und Zeus 504-5

Hera 224, 229f., 236, 563

- und Athene 237

- und die Argonauten 236

- und Erinyen 237

- und Hephaistos 229

- und Iris 238

- und Zeus 243, 254

Herakleitos
- Quaestiones Homericae 26,142 247, 428

Herakles 229, 231, 237, 243, 250, 474

Hermes 222, 237f., 251, 570, 604

- und Herse 238

Herodianos

$-1,11,1242,468$

Herodot

$-3,28,2234$

$-3,86245$

$-4,5252$

$-4,189458$

$-5,92, \eta, 1-4603$

Herrschaft 250, 252

Herrschaftskonzepte $378,384-89$

Herse 238

Hesiod 222, 430f., 441, 450

- Erga

237-246 223

- Theogonie 410-15

$117 \mathrm{f}$ und $127 \mathrm{f} 223$

123-125 246

$126 \mathrm{f} 254$

176-192 233

183-187 234, 253

195-198 234

211f. 494

211-232 246

521-569 603

$556 \mathrm{f} 603$

561-569 247

629-634 240

689241

700-705 256

758-763 494

820241

902573

Hexen 255

Hexen und Zauberer 565

Himerios

- Oratio 25249

Himmel

- als Ozean 548, 569

- an der Vorderseite des Himmels (ina pāni šamê) 184

- aus dem Himmel 60-61, 68

- aus dem Himmel . . . auf der Erde 70

- aus dem Himmelsbereich 70 
- aus dem Himmelsinneren 70

14,153 f 224

- aus dem strahlenden Himmel 69

14,225224

- aus dem strahlenden Himmelsinneren 70

14,243-262 508

- fällt auf die Erde 193, 215, 255f., 556

- Himmelsfundament 70

- Himmelsinneres 70f., 246

$14,247-261502-4$

14,319 f 227

- Himmelsinneres und An 71-73

15,16-33 509

- kommt als erster Tempel auf die Erde 556

15,18-30 231, 243

$16,431-457473$

- mit Schiffen 548

- qereb šamê (Himmelsinneres) 184

17,194-197 248

17,425222

$17,543-546237$

- zerstört die Erde 556

- zuq(q)urān šamê (Himmelshöhe) 183

Himmelsflut 226

Himmelsfundament Siehe Himmel

$18,394-405229$

18,614-617 249

18,616230

Himmelsgott Siehe An

19,91-131 474

Himmelshaus 567, 569

20,4-40 240

20,56 f 245

20,318-340 473

Himmelsklänge 244

Himmelsstimme 224

20,438-446 473

Himmelstexte 244

21,198 f 245

himmlische vs. göttliche Herkunft 260, 585, 605

Hirtenamt Siehe Numinose Machtmittel

historiolae 161, 216

21,595-598 473

$22,470-472 \quad 250$

- Odyssee

5,50238

5,294246

6,41-47 222, 224

Höhle 230, 233, 260

Homer 222

6,240-243 223

6,280 f 225

7,199225

9,69246

9,144 f 234

12,4252

12,315246

20,30 f 238

20,103 f 245

Horaz 430-31

- Carmina

1,3430

1,3,27-33 247

1,34245

$3,4,1$ f 225

- Saturae

1,5,101-103 223

Horen 222

Horus 215

Hydaspes 237

Hyginus 431-36 
- De astronomia

$$
2,13232
$$

2,15 431-36

- Fabulae

53228

144435

166231

197233

Hylem 59

- Abstraktionsgrad 47f.

- Definition 19

- Determinationen 19, 462

- duratives Hylem 23, 27

- durativ-initiales Hylem 23

- durativ-konstantes Hylem 23

- durativ-resultatives Hylem 29, 34

- dynamisches Hylem 22

- Hylemschema 48, 261, 572

- Hylemsequenz 59

- Hyperhylem 21, 28, 50, 117, 480, 568, 572

- Hyperhylemschema 572

- implizites Hylem 30

- Indeterminationsgrad 48

- isoliertes Hylem 114-17, 581

- kleinste handlungstragende Einheit 19

- konkretes Hylem 48, 117, 261

- Prädikat 19, 25, 462

- punktuelles Hylem 23

- standardisierte Darstellung 25

- Starthylem 23

- statisches Hylem 22

- Steckbrief-Hylem 23

- Zielhylem 24, 29

Hylemanalyse 18, 46, 50, 462, 464, 466

- Bestimmung von Stoffgrenzen 35

- Definition 20

- durativ-konstante Hyleme 27

- durativ-resultative Hyleme 29

- gleichzeitige Hyleme 27

- Komplettierung der Hyleme 30, 465

- Mehrwert 588

- methodische Schritte 20

- punktuelle Hyleme $27 \mathrm{ff}$.

- Rekonstruktion des Stoffverlaufs 31

- stoffchronologische Reihenfolge 27

- textliche Reihenfolge 26

Hylemelement 19, 58-59, 462
- Lokalbestimmung 68-76

- Objekt der Personenklasse 79-86

- Prädikat 61-67

- Subjekt 76-78

Hylistik 46, 50

hylistische Mythosforschung Siehe Hylistik Hypnos 224, 243

- als (Zwillings-)Bruder des Thanatos 494

- als röm. Somnus 494

- und Hera 500-502

- und Pasithea 498-99

- und Wein 497

- Unterweltsaffinität 494-95

I

Iao 215

Iasion 470

Iason 232, 247

Ida 224, 235

Iddin-Dagan 577

Ikaros 236

Ilion 461f., 472f., 475, 477, 479ff.

Ilos 241, 460, 462, 469, 472f., 479, 583, 585

Imhotep 200, 567

ina pāni šamê Siehe Himmel

Innana 563, 567, 572, 574, 577, 580ff., Siehe auch Theophanie

INNANA BRINGT DAS HIMMELSHAUS FÜR DIE ERDE

Siehe an gal karede / Innana und An

Innana raubt den großen Himmel Siehe an gal karede / Innana und An

Innana und An Siehe an gal karede / Innana und $A n$

Innana und Enki 574

Ino 237

Interhylität 581

Io 234

Iris 222, $237 f$.

Irrationalität des „Bösen“ 579

isib-Priesteramt Siehe Numinose

Machtmittel

Isis 200, 203f., 208, 210, 563

Iškur Siehe Theophanie

Iuno Siehe Hera 
Kraft 102

Jam 202

Joch 252

Julian 447

- Gegen die Kyniker 443

Jupiter 563, Siehe Zeus

Jupiter Pluvialis 206

Juvenal

- Satura 15438

K

Kadmos 21, 47, 239, 250, 478

Kallimachos

- Hymnen 4,35-38 228

Kalliope 225

Kalypso 238

Kampf um den Panzer des Inaros, P. Krall

- Kol. 1, Z. 4-1.8 197

- Kol. 9, Z.18 192

kar (sum.), rauben“

- mit Ventiv ,herbeiholen' 284-85

Kassandra 472

Kelten $255 \mathrm{f}$.

Kephalos 34

Kerberos 237

Kette 243

Keule 251

Klagen des Ipuwer

- Kol. 12, Z. 11-13 191

Kleid 251

Königtum 558, 572, 576f., 580, 585, Siehe Numinose Machtmittel

- einigende Funktion 352-53

- in Innana und Enki 343-46

- Transfer vom Himmel auf die Erde 32224, 348-49, 375-78, 379

- Transfer von Stadt zu Stadt 349-52

Koios 228

Kolaxais 252

Konon

- FGrH 26 F 1468

- FGrH 26 F 1,34 458

Konstantin 459

Konstantinopel 459
Krankheit 193, 196, 200, 215, 574, $578 f$.

Krieg 192f., 196f., 202, 205, 215

Krone Siehe Numinose Machtmittel

Kronos 233, $253 \mathrm{f}$.

Kuh des Sîn 235

Kultbild 241f., 458, 583

Kultfest 189

- Festtag 199

- Hochheben des Himmels $190 \mathrm{f}$.

- Senut-Fest 198

Kultgegenstände 258

- himmlische Herkunft 557

Kultstatue 56, $198 \mathrm{f}$.

Kulturgüter 247f., 258

- himmlische Herkunft 557

Kulturtechnik 247, 602

$\mathbf{L}$

La trouvaille de Dreheme, 1: 3162

Lagaš-Herrscherchronik

- Gegennarration zur ,Sumerischen Königsliste` 363, 365

- i 6-10 362

Laios 238

Lamaštu 174f., 180, 563, 578ff.

- Lamaštus Exil 176

Lamaštu Serie

- I 111 f 175

- II 35176

- II 163177

Lebewesen

- Abstammung vom Himmel 559

Lehre des Anchscheschonqi

- Kol. 25, Z. 12 f 191

Lehre des Ani

- P. Boulaq 4 ro, Kol. 20, Z. 14-17 198

Lehre des P. Ramesseum

- 2, Kol. 1, Z. 6210

Lehre für Merikare

- § $72 \mathrm{f} 207$

- §113, Kol. 10, Z. 8192

- §131 191

Leid 223, 576

Leiter $569 f$. 
Lemnos 230, 260

Lesses von Lampsakos 480

Libanios

- Progymnasmata 2,7,1 230

Libanon 235

Livius

$-1,20,4538$

$-5,52,7459$

$-5,54,7539-40$

- 22,1,11 246

$-22,1,12234$

$-27,37,1244$

$-27,37,2245$

$-27,37,7245$

$-28,11,2245$

$-29,14,4244$

- 40,58,6 256

Lokalbestimmung

- Herkunftsort 68-71

- Zielpunkt 74-76

Loyalistische Lehre

- § 2, 5-3, 4214

Lucanus

- 1,522-544 244

$-1,530-535245$

$-9,471-480536-37$

Lucretius

- 2,991-995 253

Ludlul bēl nēmeqi

- II 51-55 164

- II 55177

- III 70-74 164

Lugalbanda 84f., 602, Siehe auch Theophanie

- als Ama-ušumgal-ana 590

- als König 594

- als k $\mathrm{u}_{3}$ "auferstanden" 598

- als Usurpator 589, 600 f.

- himmlische Herkunft 559

- Legitimierung durch drei Rituale 599

- Machtübernahme 588

- Rückkehr aus der Unterwelt 598-99

- Unterweltsgang 590-94

Lugalbanda und Innana

- Heilige Hochzeit 590

Lugalbanda-Epos 586-601

$-1,181592$
$-1,197596$

$-2,222598$

- 2,222 (Lugalbanda wie vom Himmel) 587-88

$-2,309$ f. // 373 f. 595

$-2,319$ f. // 383 f. 596

$-2,345-355589$

- Absetzung des Herrschers 595-97

- Anzu-Vogel 594, 600

- historische Bezüge auf Utu-heĝal und UrNamma 600

- Inkubationsritual 594

- Inthronisation Enmerkaras 591

- Inthronisation Lugalbandas 592

- mit Mythos Die GötTer maCHen Lugalbanda ZUM HERRSCHER 588

- Schnelligkeit wie Gestirngötter 594

- Textoberfläche vs. Sinnebene 592

- Unterschied Text - Mythos $588 \mathrm{f}$.

Lukianos

- De sacrificiis

6231

30,8222

- Dialogi deorum 442

19,1 235

- Prometheus 439-42, 442

Lykaon 226

Lykophron

$-72-85471$

$-363485$

M

Maat 191, 573, 576

- himmlische Herkunft 558

Magier 255

Magisches Handbuch 197, 201

Magna Mater 242

Manilius

- 4,883-887 254

mannam lušpur Formel 171

Marcus Aurelius (röm. Kaiser) $205 \mathrm{f}$.

Martial

$-14,175227$

m e 567,574 , Siehe Numinose Machtmittel

Medeia 232, $251 \mathrm{f}$. 
Meer 228f., 234, 260, 572

Memnon 236

men-Krone Siehe Numinose Machtmittel

Menschen 253

- Abstammung vom Himmel 559

Mercurius Siehe Hermes

Meteorit 236, 244

Meteorit-Eisen

- himmlische Herkunft 557

Milch 244

Mond 255

Morgenröte 235

Musaios

- 109-111 236

Mutterschaf und Getreide 574

Mysterien 251

Mythenvergleiche 15, 21, 25, 31, 47ff.

- methodische Schritte 47, 49 f.

Mythische Stoffe (Auswahl)

- Agamemnon lieBt Chryseïs 41

- Apollon liebt Daphne $15 f$.

- Athene bekommt die Palladia der Groben GÖTTER 36

- Athene erhält die AIGIS 36

- Athene schenkt Chryse die Palladia 36

- DaRdanos heiratet ChRYse 26, 36, 46

- ERECHTHEUS BESIEGT EUMOLPOS 50

- GÖTTER MACHEN LUGALBANDA ZUM HERRSCHER 586-601

- GotTheit SCHICKT MENSCHEN DAS PALLADION 459-86

- GOTTHEIT SCHICKT NUMA ANCILE-SCHILD 511-35

- Hephaistos Vergewaltigt Athene 231

- HERAKLES ZERSTÖRT TROIA 501

- HeRmes eRfindet das Feuer 604

- Hypnos und HeRA tRefFen sich Auf Lemnos 493-509

- INNANA BRINGT DAS HIMMELSHAUS FÜR DIE ERDE 271-302, 569, 577, 581

- INNANA BRINGT MACHTMITTEL DER UNTERWELT ZUR ERDE 581

- NN GRÜNDET TROIA 34, 42f., 478

- Pallas zeugt Chryse 36

- Prometheus stiehlt das Feuer 409-53, 586, 601-4

- ZeUs SCHICKT DURCH HeRMES Den MENSCHEN ANSTAND UND RECHT 251
Mythische Stoffschemata (Kurztitel)

- Allophanie-Mrthen 227

- KÖNIGTUM-MYTHOS 309-89

- MENSCHENSCHÖPFUnGSMYTHOS 253

- MetamorPhosenmYthen 14

- SintflUt-MYTHOS 226, 245, 258, 471, 473, 481

- UNTERWELTSGANG-DES-KÖNIGS-MYTHOS 59094

- VERSTIRNUNGSMYTHEN 222

- Weltaltermythos 416

Mythos

- als Erzählstoff 12

- Bedeutsamkeit 576f., 580

- Definition 17

- Deutungsmachtkonflikte 39ff., 260, 485, $581 f$.

- Funktionalisierungen 576, 585

- Grundfunktion 575, 605

- hylistische Mythosforschung Siehe Hylistik

- ikonographische Darstellung 320

- Inkonsistenzen 38, 40f., 46, 471, 473, 475ff., 480, 486, 488

- Interpretation 28, 46f., 51, 476, 584, 586

- Kennzeichnung durch KAPITÄLCHEN 15

- konkrete Handlungsträger 466

- Legitimation $576 \mathrm{f}$.

- mediale Konkretionen 13, 18, 46

- Metafunktionen 578

- mythischer Dreischritt 31

- Numinosität 602, 605

- Polymorphie 13, 17, 38f., 50, 476, 482

- Polystratie 17, 38f., 476

- Prototypisches 580ff.

- Stoff vs. Stoffgestaltung 47

- teleologischer Charakter 28, 31

- Textoberfläche vs. Sinnebene 592

- Titel in Hylemform 15

- Transmedialität $12,47,50$

- und Religion 222, 602-4

- Vernetzung von Mythen 35

- Wertungen und Hierarchisierungen 40f., 51

- zielt auf Concreta, nicht Abstracta 584

Mythos von der Himmelskuh (Text) 200

- Grab Sethos' I. (KV 17) Z. 85 f 208 
N

Nacht 246, 258

Nanna/Suen Siehe Theophanie

Narrative Exemplifikation 384-85

Narratologie 13, 22, 465

Narthex-Stängel 570

Našše Siehe Theophanie

Naturphänomene 244f., 258, 261, Siehe Theophanie

Nausikaa 225

Neferti

- Z. 53 f 214

Nergal 564

Nergal und Ereš-ki-gal 569

Netz 569

Neuassyrischer Brief

- SAA 16, 63: r. 26' 173

Nike 41

Ninĝirsu 77

Ninisina 568

Nisaba 77, 567

Nonnos

- Dionysiaca

$1,87 \mathrm{f} 233$

3,376250

30,77260

35,346 f 240

38,410 f 236

42,531-533 240

48,613 f 232

nubigena 538

Numa 512, 568, 577

Numinose Machtmittel 87-93, 104-5, 561

- [...] des Königtums 88

- himmlische Herkunft 558

- Hirtenamt 87

- isib-Priesteramt 87, 90

- Königtum 87, 321-22, 371

- Krone 90 aga-Krone 87 men-Krone 88

- Schrecken(sglanz) 88

- Tafel des Lebens 91-92

- Thron 88, 90

- udug-Waffe 91, 92-93
- Zepter 88, 90

Numinoses 105

- Anzu-Vogel 98

- böse Mächte himmlischer Herkunft 554

- Feldfrüchte 100

- Getreide(gottheit) 100

- Himmelsstier 98

- Sturm und Regen 96-98

- Tiere und Pflanzen 98-101

Nymphen 234, 253

Nyx Siehe Nacht

0

Odysseus 225, 237f., 245f., 458

Okeanos 230, 245

Olymp 223f., 226, 228f., 231, 240, 245, 247f., 257, 260, 468f., 482, 573

Olympische Götter 42, 225, 232, 239f., 254f., 604

Omina 210, 213, 223, 234, 243f., 246, 258, 261

Omina brontoscopiques

- Kol. x+1, Z. x+11 213

Opfer $602 \mathrm{ff}$.

Opferbetrug 604

Orakel 189, 198f., 215

Ordnung 572, 576, 578

Orestes 241

Orientierung 578

Orphische Argonautica

- 776 f 237

Orphische Goldplättchen 254

Orphische Lithica

- 243 f 260

Orphische Schriften (Fragmente)

- 480-483 254

Ortygia 228

Osiris $193,197,564$

Othrys 240

Ovid 567

- Fasti

3,259-392 513-23

3,289-293 529-30

3,359-398 241 


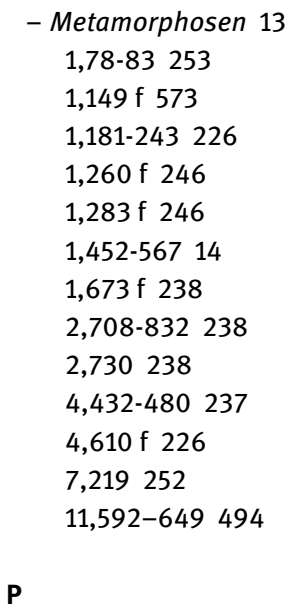

Palladia 26, 31, 41, 458, 481f., 485f., 583f.

Palladion 43, 241f., 457, 460, 464, 466, 470, 472f., 475, 481ff., 485ff., 577, $583 \mathrm{f}$.

Pallas 489

- Arkader, Vater von Chryse 26, 33, 41, 43, 481

- Arkader, Vater von Nike 41

- Gigant 41

- Titan, Vater der Athene 41

- Tochter von Triton 41, 461

Panopeus 243, 250

Papyri Demoticae Magicae, P. mag. LondonLeiden

- Kol. 20, Z. 22208

Papyri Graecae Magicae

- PGM 1, 42-195 215

- PGM 2, 80-85 215

- PGM 3, 259 f 215

- PGM 4, 1015-1020 215

- PGM 4, 1115-1160 215

- PGM 4, 1610215

- PGM 5, 460-465 215

- PGM 7, 795-845 215

- PGM 8, 64-110 215

- PGM 12, 14-95 216

- PGM 12, 96-106 216

- PGM 12, 107-121 216

- PGM 12, 201-296 216

- PGM 36, 295-311 216
Papyri Graecae Magicae, „Christliches“

- 15 a 216

- 15 b 216

Papyrus Anastasi 2 Kol. 7, Z. 6 f 212

Papyrus Anastasi 4

- Kol. 6, Z. 9, „Lob auf Piramesse“ 207

Papyrus Vandier 4, 9-16 198

Papyrus Westcar Kol. 11, Z. 10-17 209

Paris 458

Patroklos 237, 248

Pausanias 429

$-1,26,6242,458$

$-7,4,4-7242$

$-9,12,4242$

$-9,40,11$ f 250

$-10,4,4243$

Peleus 240, 248

Pelops 250, 484

Penelopeia 238

Pentheus 224

Pergamon 473

Perseus 226

Pessinus 242

Petrus Danielis 450

Pflanzen 253

Pflanzenwachstum 574

Pflug 252

Phaëthon 236

Phaiaken 225

Pherekydes von Athen 486

- fr. 3 F 97 BNJ 238

Philostratos

- Apollonios von Tyana 8,7,2 f 255

Phoibe 228

Phoroneus 428

Phrixos 238

Phylarchos

- FGrH 81 F 47483

Pindar

- Pythien

3,85-96 240

12,17 f 227

Platon

- Philebos 426-27

- Politeia 441, 445

- Protagoras 422-26 320c8-322d5 251 
- Timaios 23a f 226

Pleione 470

Plinius

- Naturalis historia

2,96 f 246

2,99 f 246

2,102244

$2,137 \quad 245$

2,138 f 245

2,146-148 244

Plot 13f., 463

Plutarch

- Fabius Maximus 2,3 244

- fr. 194a 247

- Lysias 12,1 244

- Moralia 368c 234

- Numa 13 523-31

- Timoleon 8,4-8 244

Polydoros 242

Polyneikes 238

Porphyrio 431

- ad Hor. carm. 1,3,27 und 29 f 247

Porphyrios

- De abstinentia 3,25 254

Poseidon 51, 231, 239, 245f., 251, 260

- und Alope 226

Pracht 102

Priamos 461, 468

Priester 199ff., 206, 211, 213

Priests and Officials

- 199 f(Private Collection): 27166

Proklos

- ad Plat. Tim. 1,115 236

- Bellum Gothicum 1,15 459

Prokris 34

Prolepse 14, 464, 480

Prometheen 435

Prometheus 243, 248, 253, 563, 568, 581f., $602 f$.

- als Kultstifter 602-4

- als Kulturstifter 417

- bei Diodor 427-28

- bei Platon 422-27

- Feuerdiebstahl 570,601-4

- Feuerdiebstahl auf Lemnos 420

- Prometheus-Mythen 409-53

- und Athene 449
- und Epimetheus 422, 426

- und Hephaistos 419

- und Herakles 415

- und Jupiter 431-35

- und Merkur 449

- und Thetis 451

- und Zeus 410-12, 419, 423, $441 \mathrm{f}$.

Prozession 198f., 201, $215 f$.

Pyramide 569

Pyramidentexte 569

Q

qereb apsî Siehe Abzu qereb šadê (das Innere der Berge) 184 qereb šamê Siehe Himmel

Quintus von Smyrna

$-1,118$ f 236

$-1,826$ f 236

$-2,593$ f 236

$-4,62-64236$

$-7,620-622236$

$-10,125-137235$

$-10,358-360468$

$-12,157-218240$

$\mathbf{R}$

Ramses II. 201, $207 \mathrm{ff}$.

Re 191f., 196, 199f., 209, 214, $569 f$.

Rebellen 192, 200

Recht 251, 578

Regen 205ff., 226, 244f., 253, Siehe

Numinoses

- Regen des Himmels 97

- Regensturm 563

- Überfluss 97

Rheia 254

Ritual

- Heilige Hochzeit 590

- mit Inkubation 593

- mit Unterweltsgang des Königs 590-94

- vom Unterweltsgang 1. Jt. als

Trauminkubation 593

- vom Unterweltsgang mit Heiliger Hochzeit 593 
- zur Inthronisation mit Schnelllauf 594-95

Rom 241, 459, 577

Rüstung 248, 251

\section{S}

Sachmet 196

Salier 511, 514

Samothrake 42, 470f., 473, 477, 481

Satirische Streitschrift

- §38f 214

Schafzucht 574

Schale 252

Schaum 234

Schiffbrüchiger

- Z. 125-132 195

Schild 249

Schleier 250

Schnee 205, 208

Scholien

- ad Hom. Il. 14,231 496-99

- ad Hom. Il. 15,21 243

- ad Hom. Il.16,174 467

- ad Lykophr. 29 478, 480

- ad Lykophr. 355486

Schrecken(sglanz) Siehe Numinose

Machtmittel

Schutzgötter 180

Schwelle 231

Schwert 251

Seelen 254

Seil 227, $569 f$.

Selene 234, 564

- und Endymion 235

Semele 226

Seneca

- Thyestes 789-884 255

șerretu (Leitseil) 165, 168, 181

șertu (Euter) 181

Servius

- Comm. in Verg. Buc. 447-50

Seth 194, 208f., 211, 213

Seuche 196, 578

Sextus Empiricus

- Adversus mathematicos 1,291 231

Sichel 233
Sicherheit $577 f$.

Silius Italicus

- 10,340-370 494

Sîn 234,564

Sinuhe

- Version B, Z. 43-45 196

Skarabäus 200

Skorpion 569

Skythen 252

Somnus Siehe Hypnos

Sonnenwagen 570

Sophokles

- Antigone 950227

Städte

- himmlische Herkunft 556

Statius

- Silvae

5,2,130 f. 538

- Thebais

1,197-199 238

1,303-311 238

$2,1 \mathrm{f} 238$

8,751-766 238

$10,84-155494$

Stern 228, 255

- (fallender) Stern (kakkabu) 165, 169, 182

- Stern/Komet 195f., 201, 215, 570

- Sternschnuppe 228

Stoffarten 17

Stoffpartitur $50 f$.

Stoffschema 48

Stoff-Stoff-Interferenzen 38, 40 f.

Stoffvariante 12ff., 20, 23, 465, 473, 475, $478 \mathrm{ff}$.

- Abgeschlossenheit 35, 478

- ausschnitthafter Charakter 16, 30f., 465 , 581

Story 13f., $463 \mathrm{f}$.

Strafe 223, 229, 243, 245, 256, 258, 474, 576

Stratifikationsanalyse $46,469,487,582$

- Definition 40

- methodische Schritte 40

Streitaxt 252

Sturm Siehe auch Numinoses

- Brüllen des Sturmes 97

- großer Sturm 97

- Hand des Sturmes 97 
- Sturm als Egge 97

Suda 483

Suda, Lemma 'lou入ıavós 206

Sueton

- Domitianus 15,2 245

Šulgi 576

Šulgi A Schnelllauf des Königs 595

Šulgi $P$

- Segm. B 37379

Sumer 581

,Sumerische Königsliste“ (Grundversion)

Siehe Herrschaftskonzepte, Königtum

- Beginn der Geschichte 349

- Paradigma des historischen Wandels 349-53, 377

Sumerisches Sintflut-Epos

- abhängig von der,Sumerischen Königsliste‘ 372

- CBS 10673+10867, ii 5'-7' 371

Summanus 245

$\mathbf{T}$

Tacitus

- Annalen 13,24 245

Tägliches Kultbildritual 199

Tafel des Lebens Siehe Numinose Machtmittel

Taranis 256

Targitaos 252

Tauben 233

Tempel 572, 574, 576f., 580, 582

- Aktivierung 281

- E-ana als Prototyp 302

- Himmel auf Erden 298-302

- himmlische Herkunft 556-57

- Transfer vom Himmel auf die Erde 281-83

Tempel, Stadt u. Zugehöriges 93-96, 103-4

- Berg 93

- Mauer 93

- Palast 93

- Riegel 93

- Städte 93

- Tempel 67, 94, 113-17

- Wohnsitz 94

The Cow of Sîn 171
Theben 48, 237ff., 242, 478

Themis 573

Themistios

- Oratio 8,111d 250

- Oratio 18,221a 249

Theophanie 79-86, 103

- Ama-ušumgal-ana 80

- Babu 80

- Brüllen 81

- Enmerkara 80

- Ezinam 80

- Glanz 81

- Göttlich-naturhafte Mächte 555

- Innana 80, 81-82

- Iškur 83

- Licht 81f.

- Lugalbanda 588

- Nanna/Suen 81

- Našše 80

- Naturphänomene 80

- Person 80

- Schreckensglanz 81

- Udug-Schutzgeist 80

- Utu 80

Theoxenie 239

Thetis 229, 240, 248

Thoth 191, 196, 201f., 206, 234

Thraker 256

Thron Siehe Numinose Machtmittel

Thyestes 250

Tibullus

$-1,2,43255$

$-1,8,21255$

Tiere 253, Siehe auch Numinoses

Titanen 240, $254 \mathrm{f}$.

Tore 222, 232

Transferakteure 563

Traum 237f.

Treppe 569

Tribut 202

Triton 41, 461

Troia 34, 41f., 224, 241ff., 458f., 461, 468, 470, 472, 474, 480f., 483, 485, 577, $583 f$.

Troianischer Krieg 240, 243ff., 258, 461

Troianisches Pferd 458

Tros 461, 472, 483 
Turnus 239

țurru (Band) 165, 168, 173, 181

Tydeus 238

U

Übel $223,578 f$.

udug bul 574

Udughul Vorläufer

- Ni 623+2320: vii 47 f 163

- Ni 631: i 33163

Udug-Schutzgeist Siehe Theophanie udug-Waffe Siehe Numinose Machtmittel Unsterblichkeit 238

Unterwelt 162, 176, 237f., 242, 247, 260, 572, 574, 603

Unterweltsgötter 564

Unterweltstraum des assyrischen Kronprinzen Inthronisationsritual 593

Ur 568, 576

Uranos 233f., 253f., 258

Urbild 56

Urhügel 574

Uruk 574, 577, 580, 582

Utu 77, 563f., Siehe auch Theophanie

Utu-heĝal und Ur-Namma 600

utukku lemnūtu 574

V

Venus Siehe Aphrodite

Verbannung 227, 229, 232, 241

Vergewaltigung $471 \mathrm{f}$.

Vergil 447

- Aeneis

2,162-170 458

2,692-700 244

5,606 f 239

5,835-861 494

$8,416-422249$

8,663-665 539-40

9,2239

- Eclogae

4,6 573

Verkommener Harfner

- Kol. 2, Z. 17-20 196
Versteinerung 239

Vulcanus Siehe Hephaistos

W

Wachtel 228

Wagen 251f.

Weisheitstext P. Brooklyn

- inv. 47.218.135, Kol. 3, Z. 18191

Weisheitstext $P$. Insinger

- Kol. 8, Z. 18 f 197

- Kol. 31, Z. 24-32.17 191

Weltwahrnehmung

- etische und emische Perspektiven 55961

Wenamun, P. Moskau inv. 120

- Kol. 2, Z. 13 f 211

- Kol. 2, Z. 19211

Wermai, Kol. 1Z. 6214

Wetter 205, 207f., 210f., $213 f$.

Wieland, Christoph Martin 439, 442

Wolke 205, 207f.

Z

Zepter 249f., 576, Siehe auch Numinose Machtmittel

Zeus 22, 222f., 226f., 240, 244f., 253, 258, 488, 563

- Allophanien 227

- und Alkmene 226

- und Asterië 228

- und Ate 229, 474, 579

- und Athene 237

- und Borysthenes-Tochter 252

- und Danaë 226

- und Dardanos 43, 482

- und Elektra 470ff., 477, 481

- und Erechtheus 19, 26

- und Hephaistos 230

- und Hera 243, 254

- und Hermes 238, 249, 251

- und Ilos 241, 460, 464, 467f., 473, 479, 583

- und Lykaon 226

- und Prometheus 604 
- und Themis 573

- und Titanen 240

- Wohnung 425

Zielpunkte des Himmelstransfers 549-51

- Berggipfel 550

- Erde, allgemein 549

- Inseln 550

- Könige 550-51

- Meer 550

- Schlachtfeld 550

- Städte 549

- Tempel 550

- Thronhalle 550

\section{Zikkurat 569}

- ziqqurat šamê (Tempelturm des Himmels) 183

Zivilisation

- entsteht Dank Göttern/Himmel 557

Zorn 228, 237, 243, 246, 472

Zweiter Setna-Roman, P. BM EA inv. 10822

- Kol. 5, Z. 8 f 191

- Kol. 6, Z. 4 f 213

- Kol. 6, Z. 12-15 211

- Kol. 6, Z. 24 f 213

Zwillingsgebirge 232 